American Journal of Immunology 6 (3): 43-49, 2010

ISSN 1553-619X

(C) 2010 Science Publications

\title{
Relation of Serum and Semen Malondialdehyde and Total Anti-Oxidants with Sperm Parameters in Infertile Men
}

\author{
${ }^{1}$ Firozeh Akbari-Asbagh, ${ }^{2}$ Ebrahim Mostafavi, \\ ${ }^{3}$ Kobra Hamdi, ${ }^{1}$ Ozra Azmodeh, ${ }^{1}$ Azizeh Ghasemynejad, \\ ${ }^{2}$ Jinus Moshtaghi, ${ }^{2}$ Naser Salsabili and ${ }^{4}$ Omid Mashrabi \\ ${ }^{1}$ Department of Obstetrics and Gynecology, School of Medicine, \\ Medical Sciences/University of Tehran, Tehran, Iran \\ ${ }^{2}$ Department of IVF, Mirza Kochak-Khan Hospital, \\ Medical Sciences/University of Tehran, Tehran, Iran \\ ${ }^{3}$ Department of Obstetrics and Gynecology, School of Medicine, \\ Medical Sciences/University of Tabriz, Tabriz, Iran, \\ ${ }^{4}$ General physician, Faculty of Medicine, \\ Tabriz University of Medical Sciences, Tabriz, Iran \\ Corresponding author: Mashrabi Omid, Faculty of Medicine, \\ Tabriz University of Medical Sciences, Tabriz, Iran
}

\begin{abstract}
Problem statement: Oxidative Stress (OS) has been recognized as one of the most important cause of male infertility. We studied the relation of serum and Semen Malondialdehyde (MDA) and Total Antioxidant Capacity (TAC) with sperm parameters in infertile men with sperm count within the normal range. Approach: The prospective case- control study performed on infertile men presenting to the infertility clinics of Mirzakochak khan hospital, Tehran university of Medical Sciences from June 2007 to June 2009. The samples were collected consecutively and the total of 40 infertile men was enrolled in the study. Also, 40 healthy men were matched as control group. MDA and TAC in serum and seminal plasma were measured and relation between them and semen analysis parameters were evaluated. The MDA was measured as nmol $\mathrm{mL}^{-1}$ and the TAC was expressed as $\mathrm{g}$ $\mathrm{dL}^{-1}$. Results: Analysis showed that the amount of semen MDA was statistically different in infertile and healthy control groups. We did not find any significant relation between smoking and sperm parameters in infertile men. The relation between semen MDA and abnormal sperm abnormal morphology $(\mathrm{p}=0.003, \mathrm{r}=-0.468)$ and semen TAC and weak sperm motility $(\mathrm{p}=0.037, \mathrm{r}=-0.359)$ was significant. Conclusion: Immediate attention should be directed at simplifying and validating the evaluation of reactive oxygen species and OS status so that it can be performed routinely.
\end{abstract}

Key words: Malondialdehyde (MDA), Total Antioxidant Capacity (TAC), semen analysis, parameters, morphology, Oxidative Stress (OS), validating, seminal plasma, spermatozoa, epididymis

\section{INTRODUCTION}

Infertility is a major clinical problem, affecting people medically and psychosocially. Statistics indicate that $15 \%$ of all couples in the United States are infertile and the male factor is responsible for 25 of these cases (Makker et al., 2009; Sharlip et al., 2002; Han et al., 2010). Infertility is associated with Oxidative Stress (OS), normally counterbalanced by different antioxidant systems (Mancini et al., 2009; Golbidi and Laher, 2010I). OS has been recognized as one of the most important cause of male infertility (Lanzafame et al., 2009; Desai et al., 2009; Pons-Rejraji et al., 2009; Kefer et al., 2009; Varghese et al., 2008; Tempest et al., 2008; Gallardo, 2007).

Despite the antioxidant activity of seminal plasma, epididymis and spermatozoa, OS damages sperm function and DNA integrity (Lanzafame et al., 2009; Desai et al., 2009). OS is a common pathology seen in approximately half of all infertile men. Peroxides causing infertility are generated by sperm and seminal leukocytes. Oxidative stress occurs when production of potentially destructive reactive oxygen species exceeds

Corresponding Author: Mashrabi Omid, Faculty of Medicine, Tabriz University of Medical Sciences, Tabriz, Iran Tel: +98-9144049694/+98-42262261061 
Am. J. Immunol., 6 (3): 43-49, 2010

natural antioxidant defenses resulting in cellular damage (Bozhedomov et al., 2009; Tremellen, 2008).

Malondialdehyde (MDA) is one of the final products of lipid peroxidation in seminal plasma (Shang et al., 2004). Toxic lipid peroxides are known to cause various impairments of sperm cells and may play a major role in the etiology of male infertility. Malondialdehyde (MDA) is an index of lipid peroxidation which may be a diagnostic tool for the analysis of infertility (Tavilani et al., 2005; 2008). Kumar et al. (2009) found excess ROS (Reactive oxygen species) and low antioxidant levels in the semen of infertile Oligoasthenozoospermic (OA) men. In their study, semen MDA and ROS levels of infertile group were significantly higher than their level in control group. However, antioxidants levels were significantly lower in infertile group, compared to controls.

We studied the relation of serum and semen Malondialdehyde (MDA) and Total Antioxidant Capacity (TAC) with sperm parameters in infertile men with sperm count within the normal range.

\section{MATERIALS AND METHODS}

This is a prospective case- control study performed on infertile men presenting to the infertility clinics of Mirzakochak khan hospital, Tehran University of medical science from June 2007-June 2009. The samples were collected consecutively and the total of 40 infertile men was enrolled in the study.

Inclusion criteria were the males aged $>22$ years which married for more than one year, having normal sperm count (sperm count of $\geq 20 \times 10^{6} \mathrm{~mL}^{-1}$ ) (Engel et al., 1999; Mansi et al., 2007), none were taking an oral antioxidant supplement for three months prior to the study and their wives have not been pregnant from their marriage. Exclusion criteria were intercourse problems, chronic disease including diabetes mellitus, substance, alcohol or drug abuse, semen samples with more than $1 \times 10^{6} \mathrm{~mL}^{-1}$ neutrophils using peroxidase staining (World Health Organization WHO), 1999; Marjani et al., 2008) or other round cells and use of contraception methods within last year before presentation. Also, 40 healthy men were matched about age and were selected as control group. Written informed consent was obtained from all enrollees, according to the criteria of the Ethical Committee of Tehran University of Medical Sciences.

All participants were evaluated preoperatively and the baseline information was obtained including age, weight, smoking, drinking, duration of infertility and medication. Also, clinical findings including abnormal symptoms and signs were recorded. The spermatozoid motility was expressed as grade a (rapid progressive), b (slow progressive), c (non-progressive), d (immotile) were determined using WHO standard procedures (1999) which was classified as weak (c or d) and progressive ( $a$ or $b$ ).

All semen samples were collected by masturbation following 3 days of abstinence. The blood and semen sampling was followed by their plasma isolation. The semen and blood plasma were stored in $-20^{\circ} \mathrm{C}$. Then, Malondialdehyde (MDA) and Total Anti-oxidant Capacity (TAC) of them were measured every 6 months. The MDA was measured as nmol $\mathrm{mL}^{-1}$ and the TAC measured as $\mathrm{g} \mathrm{dL}^{-1}$.

Measurement of TAC levels: The total antioxidant status of the plasma was measured using a novel automated direct measurement method using a new generation, more stable ABTS (2,2-azinobis 3ethylbenzothiazoline-6-sulfate) radical action (Erel, 2004; Xiaoming et al., 2007)). In novel methods, a type of radical is generated in the assay and the antioxidant activity of the sample against the radical is measured. The most common method is ABTS-based method in which a colorless molecule, reduced ABTS, is oxidized to a blue-green ABTS. When the colored ABTS is mixed with any substrate that can be oxidized, it is reduced to its original colorless form again (Erel, 2004; Mansi et al., 2007).

Measurement of MDA levels: Lipid peroxidation in spermatozoa and seminal plasma was measured by reaction of Thiobarbiuric Acid (TBA) with Malondialdehyde (MDA) (Tavilani et al., 2008). Content of MDA was measured spectrofluorometrically using a Jasco (FP-6200) spectrofluorometer (excitation $515 \mathrm{~nm}$, emission $553 \mathrm{~nm}$ ). The MDA fluorescence intensity of spermatozoa and seminal plasma was determined using various concentrations of tetraethoxypropane as standards. The results were expressed as nmoL MDA $/ 10 \times 10^{6}$ cells, nmoL MDA $\mathrm{mL}^{-1}$ seminal plasma and nmoL MDA/total seminal plasma.

Statistical analysis: The collected data were analyzed by SPSS-15 statistical software. The collected data were expressed as percentage and mean \pm SD. Continuous (quantitative) variables were compared by Student T-test (Independent samples) or ANOVA one way. Categorical (qualitative) variables were compared by contingency tables and Chi-square test or Fisher's exact test. P-value $\leq 0.05$ was considered statistically significant.

\section{RESULTS}

The average age was 33.7 year (range: $32.5-35 y$ ) in infertile (case group) and 34.7 years (range: $32.5-37 y$ ) in healthy men (control group) ( $\mathrm{p}>0.05)$. As showed in Table 1, the patients' job was not significantly different in both groups. 
Am. J. Immunol., 6 (3): 43-49, 2010

Table 1: Comparison of the patients' job in both groups

\begin{tabular}{|c|c|c|c|c|}
\hline \multirow[b]{2}{*}{ Group } & \multicolumn{4}{|c|}{ Occupation } \\
\hline & \multicolumn{2}{|l|}{ Free } & \multicolumn{2}{|l|}{ Worker } \\
\hline Infertile (Case) & \multicolumn{2}{|l|}{$4(12.1 \%)$} & \multicolumn{2}{|l|}{$10(30.3 \%)$} \\
\hline Fertile (Control) & \multicolumn{2}{|l|}{$0(0 \%)$} & \multicolumn{2}{|l|}{$9(33.3 \%)$} \\
\hline & Group & Mean & SD & p-value \\
\hline \multirow[t]{2}{*}{ Serum MDA } & Infertile & 3.4551 & 1.16502 & $\frac{<0.05}{<}$ \\
\hline & Fertile & 3.2318 & 0.94052 & \\
\hline \multirow[t]{2}{*}{ Serum TAC } & Infertile & 3.0259 & 0.69407 & $<0.05$ \\
\hline & Fertile & 3.1755 & 0.61584 & \\
\hline \multirow[t]{2}{*}{ Semen MDA } & Infertile & 3.0946 & 0.84501 & 0.013 \\
\hline & Fertile & 2.6068 & 0.86426 & \\
\hline \multirow[t]{2}{*}{ Semen TAC } & Infertile & 4.5521 & 1.04654 & $<0.05$ \\
\hline & Fertile & 4.8110 & 0.75968 & \\
\hline
\end{tabular}

Table 3:The effect of cigarette smoking on serum and semen variables

\begin{tabular}{lllll}
\hline & Smoking & Mean & SD & p-value \\
\hline Serum MDA & No & 3.3430 & 1.15580 & $<0.05$ \\
& Yes & 3.6864 & 1.28447 & \\
Serum TAC & No & 2.9804 & 0.66664 & $<0.05$ \\
& Yes & 3.1791 & 0.79113 & \\
Semen MDA & No & 3.2487 & 0.89758 & $<0.05$ \\
& Yes & 2.9345 & 0.85535 & \\
Semen TAC & No & 4.4683 & 1.16991 & $<0.05$ \\
& Yes & 4.8455 & 0.98257 & \\
\hline
\end{tabular}

The mean values of serum and plasma MDA and TAC are showed in Table 2. Analysis showed that only the amount of semen MDA was statistically different in both groups (Table 2).

Table 3 shows the effect of cigarette smoking on serum and semen variables in infertile men. Analysis did not show any significant relation between smoking and studied variables in infertile men (Table 3 ).

We studied the relation of serum and semen MDA and TAC level with variables including sperm morphology, motility grade, duration of infertility and patient occupation. As showed in Table 4, only the relation between semen MDA and abnormal sperm morphology $(\mathrm{p}=0.003, \mathrm{r}=-0.468)$ and semen TAC and weak motility $(\mathrm{p}=0.037, \mathrm{r}=-0.359)$ was significant.

\section{DISCUSSION}

The male factor is considered a major contributory factor to infertility. Apart from the conventional causes for male infertility such as varicocele, cryptorchidism, infections, obstructive lesions, cystic fibrosis, trauma and tumors, a new and important cause has been identified: oxidative stress ((Makker et al., 2009). Oxidative Stress (OS) is a result of the imbalance between Reactive Oxygen Species (ROS) and antioxidants in the body. It is a major etiological cause of

sperm damage, deformity and eventually, male infertility (Makker et al., 2009; Agarwal et al., 2009). OS is a common pathology seen in approximately half of all infertile men. Increased Radical Oxidative Species (ROS) generation and reduced antioxidant capacity is negatively correlated with sperm concentration and motility in infertile men (El-Taieb et al., 2009).

OS is induced by Reactive Oxygen Species (ROS), or free radicals and although ROS are required for critical aspects of sperm function, excessive levels of ROS can negatively impact sperm quality (Kefer et al., 2009). ROS have an important effect on sperm quality and quantity. Oxidative Stress (OS) occurs when production of potentially destructive ROS exceeds the body's own natural antioxidant defenses, resulting in cellular damage (El-Taieb et al., 2009). Aydemir et al. suggest that increased oxidative damage might be a factor for hyperviscosity of seminal plasma in infertile males (Aydemir et al., 2008).

Lipid peroxidation has a deleterious effect on the semen quality and may play a major role in the etiology of male infertility (Tavilani et al., 2005; 2008). Malondialdehyde can be used as a marker of oxidative stress and a potential marker in predicting assisted reproductive techniques outcome (Oral et al., 2006; Jedrzejczak et al., 2005). One can determine the level of lipid peroxidation as indicated by in the seminal plasma (Tavilani et al., 2005; 2008). The deleterious effect of free radicals on spermatozoa may be assessed by estimating malondialdehyde in seminal plasma and their relation with different sperm parameters (Patel et al., 2009). Patel et al. (2009) analyzed 22 fertile controls with 74 primary infertile males. Seminal malondialdehyde level was observed to be raised in all infertile groups except azoospermic cases in comparison to control. Significant positive correlation between malondialdehyde with total sperm count indicates its contribution towards free radical generation. The negative association of semen malondialdehyde with normal sperm motility and morphology suggests damaging effect of free radicals on sperm membrane integrity (Patel et al., 2009). In agreement with these studies, our findings show the relation between semen MDA and abnormal sperm abnormal morphology $(p=0.003, r=-0.468)$ and semen TAC and weak sperm motility $(\mathrm{p}=0.037, \mathrm{r}=$ $0.359)$ was significant. 
Am. J. Immunol., 6 (3): 43-49, 2010

Table 4: Assessment of relationships in infertile group

\begin{tabular}{|c|c|c|c|c|c|c|c|c|}
\hline & \multicolumn{2}{|c|}{ Serum MDA } & \multicolumn{2}{|c|}{ Serum TAC } & \multicolumn{2}{|c|}{ Semen MDA } & \multicolumn{2}{|c|}{ Semen TAC } \\
\hline & $\mathrm{r}$ & $\mathrm{p}$-value & $\mathrm{r}$ & p-value & $\mathrm{r}$ & p-value & $\mathrm{r}$ & p-value \\
\hline Abnormal Morphology & 0.049 & 0.768 & 0.075 & 0.652 & -0.468 & 0.003 & -0.287 & 0.076 \\
\hline Weak motility Grade d & -0.161 & 0.228 & -0.221 & 0.095 & 0.075 & 0.574 & -0.124 & 0.352 \\
\hline Weak motility Grade c & 0.098 & 0.581 & -0.083 & 0.642 & 0.129 & 0.466 & -0.359 & 0.037 \\
\hline Progressive Motility (Grade b) & 0.447 & 0.082 & -0.017 & 0.951 & 0.396 & 0.129 & 0.054 & 0.843 \\
\hline Progressive Motility (Grade a) & $?$ & $?$ & $?$ & $?$ & $?$ & $?$ & $?$ & $?$ \\
\hline Duration of infertility & 0.067 & 0.694 & -0.178 & 0.291 & -0.114 & 0.503 & -0.025 & 0.884 \\
\hline Patient Occupation & $?$ & $?$ & $?$ & $?$ & $?$ & $?$ & $?$ & $?$ \\
\hline
\end{tabular}

Giulini et al. (2009) found no significant differences in serum TAC (Total antioxidant capacity) between controls and groups (varicocele cases). In patients with varicocele and moderate or severe oligoasthenozoospermia, seminal TAC was significantly lower than controls and normozoospermic patients with varicocele. Moreover, in patients with severe oligosthenozoospermia, seminal TAC was also significantly lower than in asthenozoozpermic patients with varicocele. In all subjects, seminal TAC showed a positive correlation with sperm concentration and motility (Giulini et al., 2009). One study showed that TAC was negatively related to sperm concentration. However, there was no significant relationship between hormone concentrations, sperm DNA damage and total antioxidant capacity, suggesting other mechanisms for sperm dysfunction (Appasamy et al., 2007).

Taking into account the pros and the cons of antioxidant treatment of male infertility, the potential advantages that it offers cannot be ignored. Therefore, antioxidant therapy should remain in the forefront of preventive medicine, including human reproductive medicine (Lanzafame et al., 2009). Because oxidative stress is a major cause of sperm DNA damage then antioxidants should have an important therapeutic role to play in the clinical management of male infertility (World Health Organization (WHO), 1999; Ismail et al., 2009). Study of aspects of oxidative stress help us to design better antioxidant trials in future, with emphasis on identifying (Makker et al., 2009) appropriate doses (Sharlip et al., 2002; Han et al., 2010) selecting the right populations ((Mancini et al., 2009) treating for optimal durations and (Golbidi and Laher, 2010) specific intracellular targeting mechanisms (Golbidi and Laher, 2010). Understanding the physiologic and pathologic effects of free radicals on sperm function will help in designing new and effective treatment strategies in male infertility (Jedrzejczak et al., 2005).

Reactive Oxygen Species (ROS) levels in semen are believed to play both physiological and pathological roles in male fertility (Venkatesh et al., 2009). Increased ROS levels also have been correlated with decreased sperm motility. However, the exact mechanism through which ROS causes decreased motility is not understood (Makker et al., 2009). Not only do antioxidants prevent reduction in sperm motility, these also increase sperm motility (Makker et al., 2009). A randomized double-blind controlled trial has shown that vitamin $\mathrm{E}$ administered orally $\left(300 \mathrm{mg} \mathrm{day}{ }^{-1}\right.$ ) results in a decrease in malondialdehyde (a) marker for lipid peroxidation) concentration in spermatozoa and improved sperm motility (Suleiman et al., 1996; Ismail et al., 2009). Elevated ROS levels in the idiopathic Indian infertile men is one of the underlying reasons for impaired fertility. Therefore measurement of seminal oxidative status may be used in infertile men for better understanding of the etiology and selection of antioxidant regimen in the treatment of male infertility (Venkatesh et al., 2009).

Tobacco smoke consists of approximately 4,000 compounds such as alkaloids, nitrosamines and inorganic molecules and many of these substances are reactive oxygen or nitrogen species. Significant positive association has been reported between active smoking and sperm DNA fragmentation, as well as axonemal damage and decreased sperm count. Levels of DNA strand breaks (Makker et al., 2009). In a study carried out on 655 smokers and 1131 non smokers, cigarette smoking was associated with a significant decrease in sperm density (-15.3\%), total sperm count (-17.5\%) and total number of motile sperm (-16.6\%) (Künzle et al., 2003; Ismail et al., 2009). Thus, smoking can affect the quality and quantity of sperm present within a male (Makker et al., 2009). However, we did not find any significant relation between smoking and sperm parameters in infertile men.

Oxidative stress is now recognized as a common pathology that affects up to half of all infertile men. One of the principal mechanisms by which oxidative stress produces infertility is by damage to sperm DNA, either through direct oxidation of the DNA by ROS or by the initiation of apoptosis. Antioxidant therapy resulted in significant improvements in sperm DNA integrity, accompanied by a reduction in seminal ROS production and apoptosis (Tunc et al., 2009). OS, 
Am. J. Immunol., 6 (3): 43-49, 2010

characterized by increased free radicals, may lead to oxidation of nucleotides of sperm genome. Shamsi et al. (2009) study, increased malondialdehyde (a product of lipidperoxides) levels, abnormal sperm morphology and higher DNA damage were observed in infertile men. The antioxidants superoxide dismutase, catalase and glutathione had a positive association with sperm count and motility while a negative association with the percentage of dead sperms and abnormal morphology was observed (Shamsi et al., 2009).

Mostafa et al. (2009) compared two ROS parameters (malondialdehyde, hydrogen peroxide) and five antioxidants (superoxide dismutase, catalase, glutathione peroxidase, vitamin $\mathrm{E}$ and vitamin $\mathrm{C}$ ) in the seminal plasma of fertile and infertile men. Compared with fertile healthy men, in all other studied groups, estimated seminal ROS were significantly higher and estimated antioxidants were significantly lower. Sperm concentration, total sperm motility as well as sperm normal forms were negatively correlated with seminal malondialdehyde and were positively correlated with vitamin C (Mostafa et al., 2009).

Pasqualotto et al. (2008) used ROS-TAC score to compare the semen quality score and the seminal oxidative stress Reactive Oxygen Species (ROS) and total antioxidant capacity in men with idiopathic infertility. They concluded that patients with idiopathic infertility have lower scores of semen quality and ROSTAC (Pasqualotto et al., 2008).

Mahfouz et al. suggested that Total Antioxidant Capacity (TAC) of the seminal plasma as measured by the colorimetric assay is a reliable and simple test for the diagnosis and management of male infertility. They showed that the best cutoff to distinguish between fertile controls and infertile men is $1420 \mu \mathrm{M}$ of TAC in the seminal plasma (Mahfouz et al., 2009). Microscopically abnormal semen showed significantly higher levels of MDA in seminal plasma as compared with normal semen. The percentage of non-motile spermatozoa showed significant positive correlation with MDA. Sperm counts showed a significant negative correlation with MDA level of seminal plasma. MDA of $3.15 \mu \mathrm{mol}$ $\mathrm{L}^{-1}$ is an optimum cut-off limits to discriminate abnormal semen from normal (Saraniya et al., 2008).

Tavilani et al. (2008) found positive correlations between TAC with total content of MDA in seminal plasma from normozoospermic samples. However, in asthenozoospermic samples, there were no such significant correlations. These findings indicate a protective role for antioxidant enzymes of seminal plasma against lipid peroxidation of spermatozoa in normozoospermic samples (Tavilani et al., 2008). Abdallah et al. (2009) found that seminal MDA was significantly higher in oligoasthenozoospermic and asthenozoospermic groups compared with normozoospermic group. Seminal MDA were positively correlated with mobility grade $\mathrm{b}$, acrosome anomalies and residual cytoplasmic droplets. In contrast, seminal MDA was negatively correlated with sperm concentrations (Abdallah et al., 2009).

Studies suggested that detection of MDA concentrations in seminal plasma has an indicative value on the diagnosis of male infertility induced by overproduction of reactive oxygen species in male reproductive system (Shang et al., 2004). The evaluation of oxidative status and antioxidant defenses may be taken as an important tool for diagnosis and treatment of male infertility (Abdallah et al., 2009). The measurement of the antioxidative and oxidative agents could serve to evaluate human infertility in those cases where the result of the spematobioscopy appears normal (Gallardo, 2007). Although consensus is growing about the clinical utility of seminal oxidative stress testing in infertility clinics, standardization of protocols to measure ROS is crucial before introducing these tests into routine clinical practice (Deepinder et al., 2008).

\section{CONCLUSION}

Evaluation of OS status and the use of antioxidants is not a routine in clinical practice. Immediate attention should be directed at simplifying and validating the evaluation of reactive oxygen species and OS status so that it can be performed routinely. The dose and duration of therapeutic antioxidants should also be determined and standardized.

\section{REFERENCES}

Abdallah, F.B., I. Dammak, H. Attia, B. Hentati and L. Ammar-Keskes, 2009. Lipid peroxidation and antioxidant enzyme activities in infertile men: Correlation with semen parameter. J. Clin. Lab. Anal., 23: 99-104. DOI: 10.1002/jcla.20297

Agarwal, A., A.C. Varghese and R.K. Sharma, 2009. Markers of oxidative stress and sperm chromatin integrity. Methods Mol. Biol., 590: 377-402. DOI: 10.1007/978-1-60327-378-7_24

Appasamy, M., S. Muttukrishna, A.R. Pizzey, O. Ozturk and N.P. Groome et al., 2007. Relationship between male reproductive hormones, sperm DNA damage and markers of oxidative stress in infertility. Reprod Biomed. Online, 14: 159-65. DOI: 10.1016/S1472-6483(10)60783-3 
Am. J. Immunol., 6 (3): 43-49, 2010

Aydemir, B., I. Onaran, A.R. Kiziler, B. Alici and M.C. Akyolcu, 2008. The influence of oxidative damage on viscosity of seminal fluid in infertile men. J. Androl., 29: 41-6. DOI: 10.2164/jandrol.107.003046

Bozhedomov, V.A., D.S. Gromenko, I.V. Ushakova, M.V. Toroptseva and S.N. Galimov et al., 2009. Oxidative stress of spermatozoa in pathogenesis of male infertility. Urologiia, 2: 51-56. PMID: 19526875

Deepinder, F., M. Cocuzza and A. Agarwal, 2008. Should seminal oxidative stress measurement be offered routinely to men presenting for infertility evaluation? Endocr Pract, 14: 484-491. PMID: 18558605

Desai, N., E. Sabanegh Jr, T. Kim and A. Agarwal, 2009. Free radical theory of aging: Implications in male infertility. Urology, 75: 14-19. DOI: 10.1016/j.urology.2009.05.025

El-Taieb, M.A.A., R. Herwig, E.A. Nada, J. Greilberger and M. Marberger, 2009. Oxidative stress and epididymal sperm transport, motility and morphological defects. Eur. J. Obstet Gynecol. Reprod Biol., 144: S199-S203. DOI: 10.1016/j.ejogrb.2009.02.018

Engel, S., T. Schreiner and R. Petzoldt, 1999. Lipid peroxidation in human spermatozoa and maintenance of progressive sperm motility. Andrologia, 3: 17-22. DOI: $10.1111 / \mathrm{j} .1439-$ 0272.1999.tb02837.x

Erel, O., 2004. A novel automated direct measurement method for total antioxidant capacity using a new generation, more stable ABTS radical cation. Clin. Biochem., 37: 277-285. PMID: 15003729 DOI: 10.1016/j.clinbiochem.2003.11.015

Gallardo, J.M., 2007. Evaluation of antioxidant system in normal semen. Rev. Invest. Clin., 59: 42-47. PMID: 17569299

Giulini, S., V. Sblendorio, S. Xella, A. La Marca and B. Palmieri et al., 2009. Seminal plasma total antioxidant capacity and semen parameters in patients with varicocele. Reprod Biomed. Online, 18: 617-621. PMID: 19549438

Golbidi, S. and I. Laher, 2010. Antioxidant therapy in human endocrine disorders. Med. Sci. Monit., 16: 9-24. PMID: 20037503

Han, N.M. and C.Y. May, 2010. Determination of antioxidants in oil palm leaves (elaeis guineensis). Am. J. Applied Sci., 7: 1243-1247. DOI: 10.3844/ajassp.2010.1243.1247

Ismail, B.Z., O.M. AL-Ababneh and M.B. AL-Zghoul, 2009. Serum levels of insulin-like growth factor-i, thyroid hormones and skeletal muscle fiber size in castrated lambs with and without androgen treatment. Am. J. Applied Sci., 6: 518-522. DOI: 10.3844/ajassp.2009.518.522
Jedrzejczak, P., M.F. Czek , S.K. Kol, G. Taszarek-Hauke and L. Pawelczyk et al., 2005. Consequences of semen inflammation and lipid peroxidation on fertilization capacity of spermatozoa in in vitro conditions. Int. J. Androl., 28: 275-283. DOI: $10.1111 / \mathrm{j} .1365-2605.2005 .00547 . x$

Kefer, J.C., A. Agarwal and E. Sabanegh, 2009. Role of antioxidants in the treatment of male infertility. Int. J. Urol., 16: 449-457. PMID: 19383039

Kumar, R., S. Venkatesh, M. Kumar, M. Tanwar and M.B. Shasmsi et al., 2009. Oxidative stress and sperm mitochondrial DNA mutation in idiopathic oligoasthenozoospermic men. Indian J. Biochem. Biophys., 46: 172-177.

Künzle, R., M.D. Mueller, W. Hänggi, M.H. Birkhäuser and H. Drescher et al., 2003. Semen quality of male smokers and nonsmokers in infertile couples. Fertil Steril, 79: 287-291. DOI: 10.1016/S00150282(02)04664-2

Lanzafame, F.M., S.L. Vignera, E. Vicari and A.E. Calogero, 2009. Oxidative stress and medical antioxidant treatment in male infertility. Reprod Biomed. Online, 19: 638-659. DOI: 10.1016/j.rbmo.2009.09.014

Mahfouz, R., R. Sharma, D. Sharma, E. Sabanegh and A. Agarwal, 2009. Diagnostic value of the Total Antioxidant Capacity (TAC) in human seminal plasma. Fertil Steril, 91: 805-811. DOI: 10.1016/j.fertnstert.2008.01.022

Makker, K., A. Agarwal and R. Sharma, 2009. Oxidative stress and male infertility. Indian J. Med. Res., 129: 357-367.

Mancini, A., R. Festa, A. Silvestrini, N. Nicolotti and V. Di Donna et al., 2009. Hormonal regulation of total antioxidant capacity in seminal plasma. J. Androl., 30: 534-540. DOI: 10.2164/jandrol.108.006148

Mansi, K.M.S., 2007. Study the effects of ramadan fasting on the serum glucose and lipid profile among healthy Jordanian students. Am. J. Applied Sci., 4: 565-569. DOI: 10.3844/ajassp.2007.565.569

Marjani, A., 2008. Effect of storage time and temperature on serum analytes. Am. J. Applied Sci., 5: 1047-1051. DOI:10.3844/ajassp.2008.1047.1051

Mostafa, T., T. Anis, H. Imam, A.R. El-Nashar and I.A. Osman, 2009. Seminal reactive oxygen species-antioxidant relationship in fertile males with and without varicocele. Andrologia, 41: 125-129. DOI: $10.1111 /$ j.1439-0272.2008.00900.x 
Am. J. Immunol., 6 (3): 43-49, 2010

Oral, O., T. Kutlu, E. Aksoy, C. Fiçicioğlu and H. Uslu et al., 2006. The effects of oxidative stress on outcomes of assisted reproductive techniques. J. Assist Reprod Genet., 23: 81-85. PMID: 16391897

Pasqualotto, F.F., R.K. Sharma, E.B. Pasqualotto and A. Agarwal, 2008. Poor semen quality and ROSTAC scores in patients with idiopathic infertility. Urol Int., 81: 263-270. DOI: 10.1159/00 PMID: 18931540

Patel, S., S. Panda, R. Nanda, M. Mangaraj and P.C. Mohapatra, 2009. Influence of oxidants and anti-oxidants on semen parameters in infertile males. J. Indian Med. Assoc., 107: 78-80. PMID: 19585814

Pons-Rejraji, H., B. Sion, F. Saez, F. Brugnon and L. Janny et al., 2009. Role of Reactive Oxygen Species (ROS) on human spermatozoa and male infertility. Gynecol Obstet Fertil, 37: 529-635. PMID: 19481966

Saraniya, A., B.C. Koner, P. Doureradjou, N. Selvaraj and V. Shivagourou, 2008. Altered malondialdehyde, protein carbonyl and sialic acid levels in seminal plasma of microscopically abnormal semen. Andrologia, 40: 56-57. DOI: $10.1111 / \mathrm{j} .1439-0272.2008 .00811 . x \quad$ PMID: 18211303

Shamsi, M.B., S. Venkatesh, M. Tanwar, P. Talwar and R.K. Sharma et al., 2009. DNA integrity and semen quality in men with low seminal antioxidant levels. Mutat Res., 665: 29-36. DOI: 10.1016/j.mrfmmm.2009.02.017

Shang, X.J., K. Li , Z.Q. Ye, Y.G. Chen and X. Yu et al., 2004. Analysis of lipid peroxidative levels in seminal plasma of infertile men by highperformance liquid chromatography. Arch. Androl., $\quad 50$ : $\quad 411-416 . \quad$ DOI: 10.1080/01485010490484138

Sharlip, I.D., J.P. Jarow, A.M. Belker, L.I. Lipshultz and M. Sigman et al., 2002. Best practice policies for male infertility. Fertil Steril, 77: 873-882. PMID: 12009338

Suleiman, S.A., M.E. Ali, Z.M. Zaki, E.M. el-Malik and M.A. Nasr 1996. Lipid peroxidation and human sperm motility: protective role of vitamin $\mathrm{E}$. J. Androl, 17: 530-537. PMID: 8957697

Tavilani, H., M. Doosti and H. Saeidi, 2005. Malondialdehyde levels in sperm and seminal plasma of asthenozoospermic and its relationship with semen parameters. Clin. Chim. Acta, 356: 199203. PMID: 15936318 DOI: 10.1016/j.ccen.2005.01.017
Tavilani, H., M.T. Goodarzi, A. Vaisi-raygani, S. Salimi and T. Hassanzadeh, 2008. Activity of antioxidant enzymes in seminal plasma and their relationship with lipid peroxidation of spermatozoa. Int. Braz. J. Urol., 34: 485-491. PMID: 18778500

Tempest, H.G., S.T. Homa , E.J. Routledge , A. Garner and X.P. Zhai et al., 2008. Plants used in Chinese medicine for the treatment of male infertility possess antioxidant and anti-oestrogenic activity. Syst. Biol. Reprod. Med., 54: 185-195. PMID: 18942026

Tremellen, K., 2008. Oxidative stress and male infertility-a clinical perspective. Hum. Reprod. Update, 14: 243-258. DOI: 10.1093/humupd/dmn004

Tunc, O., J. Thompson and K. Tremellen, 2009. Improvement in sperm DNA quality using an oral antioxidant therapy. Reprod Biomed Online.18: 761-768. PMID: 19490779

Varghese, A.C., S.S. du Plessis and A. Agarwal, 2008. Male gamete survival at stake: Causes and solutions. Reprod. Biomed. Online, 17: 866-880. PMID: 19079972

Venkatesh, S., A.M. Riyaz, M.B. Shamsi, R. Kumar and N.P. Gupta et al., 2009. Clinical significance of reactive oxygen species in semen of infertile Indian men. Andrologia, 41: 251-256. PMID: 19601938

World Health Organization (WHO), 1999. Laboratory Manual for the Examination of Human Semen and Semen-Cervical Mucus Interactio., 4th Edn., Cambridge University Press, USA., pp: 138.

Xiaoming, L., S. Wanxiang, A.T. Khalaf, S. Jiquan and T. Jinquan, 2007. Relation between autoimmunity chronic urticaria and the levels of plasma prothrombin F1+2. Am. J. Applied Sci., 4: 965-969. DOI:10.3844/ajassp.2007.965.969 